From the National Cancer Institute, National Institutes of Health, Bethesda, $\mathrm{MD}$; University of California at San Francisco, San Francisco, CA; University of Texas M. D. Anderson Cancer Center. Houston; and Southwestern Medical Center, Dallas, TX; Washington University School of Medicine, St Louis, MO; Memorial Sloan-Kettering Cancer Center, New York, NY; Yale University School of Medicine, New Haven, CT; AstraZeneca, Wilmington, DE; and Institut Gustave Roussy, Villejuif, France.

Submitted May 3, 2009; accepted October 19, 2009; published online ahead of print at www.jco.org on January 11, 2010.

Supported financially by AstraZeneca.

Presented in part at the 32nd Annual Meeting of the European Thyroid Association, September 1-5, 2007, Leipzig. Germany; the 43rd Annual Meeting of the American Society of Clinical Oncology, June 1-5, 2007, Chicago, IL; the 42nd Annual Meeting of the American Society of Clinical Oncology, June 2-6, 2006 Atlanta, GA; and the 88th Annual Meeting of the Endocrine Society, June 24-27, 2006, Boston, MA.

Authors' disclosures of potential conflicts of interest and author contributions are found at the end of this article.

Clinical Trials repository link available on JCO.org.

Corresponding author: Samuel A. Wells Jr, MD, Dept of Surgery, Washington University School of Medicine, Box 8109, 660 S Euclid Ave, St Louis, MO 63110; e-mail:wellss@wudosis.wustl .edu

๑) 2010 by American Society of Clinical Oncology

0732-183X/10/2805-767/\$20.00

DOI: $10.1200 / \mathrm{JCO} .2009 .23 .6604$

\title{
Vandetanib for the Treatment of Patients With Locally Advanced or Metastatic Hereditary Medullary Thyroid Cancer
}

Samuel A. Wells Jr, Jessica E. Gosnell, Robert F. Gagel, Jeffrey Moley, David Pfister, Julie A. Sosa, Michael Skinner, Annetta Krebs, James Vasselli, and Martin Schlumberger

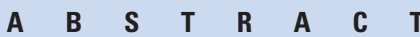

\section{Purpose}

There is no effective therapy for patients with distant metastasis of medullary thyroid carcinoma (MTC). Activating mutations in the RET proto-oncogene cause hereditary MTC, which provides a strong therapeutic rationale for targeting RET kinase activity. This open-label, phase II study assessed the efficacy of vandetanib, a selective oral inhibitor of RET, vascular endothelial growth factor receptor, and epidermal growth factor receptor signaling, in patients with advanced hereditary MTC.

\section{Methods}

Patients with unresectable locally advanced or metastatic hereditary MTC received initial treatment with once-daily oral vandetanib $300 \mathrm{mg}$. The dose was adjusted additionally in some patients on the basis of observed toxicity until disease progression or any other withdrawal criterion was met. The primary assessment was objective tumor response (by RECIST [Response Evaluation Criteria in Solid Tumors]).

\section{Results}

Thirty patients received initial treatment with vandetanib $300 \mathrm{mg} / \mathrm{d}$. On the basis of investigator assessments, $20 \%$ of patients (ie, six of 30 patients) experienced a confirmed partial response (median duration of response at data cutoff, 10.2 months). An additional 53\% of patients (ie, 16 of 30 patients) experienced stable disease at $\geq 24$ weeks, which yielded a disease control rate of $73 \%$ (ie, 22 of 30 patients). In 24 patients, serum calcitonin levels showed a 50\% or greater decrease from baseline that was maintained for at least 4 weeks; 16 patients showed a similar reduction in serum carcinoembryonic antigen levels. The most common adverse events were diarrhea (70\%), rash (67\%), fatigue (63\%), and nausea $(63 \%)$

\section{Conclusion}

In this study, vandetanib demonstrated durable objective partial responses and disease control with a manageable adverse event profile. These results demonstrate that vandetanib may provide an effective therapeutic option in patients with advanced hereditary MTC, a rare disease for which there has been no effective therapy.

\section{J Clin Oncol 28:767-772. (C) 2010 by American Society of Clinical Oncology}

\section{INTRODUCTION}

Medullary thyroid carcinoma (MTC), a malignancy of the parafollicular C cells of the thyroid, accounts for up to $10 \%$ of all thyroid cancers. ${ }^{1}$ MTC presents in sporadic form (75\% of occurrences) or hereditary form (25\% of occurrences), and the latter includes three distinct inherited cancer syndromes: familial MTC (FMTC), multiple endocrine neoplasia (MEN) type 2A (MEN2A), and MEN2B. ${ }^{2}$ The prognosis is generally favorable if the disease is treated at an early stage, as the 10 -year survival rate is $70 \%$ to $80 \%{ }^{3}$ However, 10 -year survival rates are less than $50 \%$ in patients with distant metastatic disease. ${ }^{3}$ External-beam radiation therapy has a limited role in patients with advanced MTC, and conventional cytotoxic chemotherapy has not been proven to prolong survival. ${ }^{4}$

Germline mutations in the RET (ie, REarranged during Transfection) proto-oncogene cause hereditary MTC, and up to $50 \%$ of patients with sporadic MTC have somatic RET mutations. ${ }^{4-6}$ The constitutively active RET oncoproteins associated with MTC have been shown to be highly sensitive to agents that inhibit wild-type RET kinase, which thereby provides a strong rationale for targeting RET in patients with MTC. ${ }^{7}$ In addition, thyroid tumors are vascular, and increased expression of 
vascular endothelial growth factor (VEGF) is associated with increased tumor growth and invasiveness. ${ }^{8}$ Inhibitors of the VEGF receptor (VEGFR), therefore, may have antitumor activity in MTC.

Vandetanib (ZACTIMA; AstraZeneca, Wilmington, DE) is a once-daily oral agent that selectively targets RET-dependent, VEGFRdependent, and epidermal growth factor receptor (EGFR)-dependent signaling. ${ }^{7,9}$ Vandetanib has been shown to inhibit tumor cell proliferation in a broad range of preclinical models. ${ }^{9-11}$ Phase I evaluation in patients with solid tumors showed that vandetanib monotherapy (up to $300 \mathrm{mg} / \mathrm{d}$ ) was generally well tolerated and that the pharmacokinetic profile supported a once-daily oral dosing regimen. ${ }^{12,13}$ In this study, we evaluated the efficacy and tolerability of vandetanib in an open-label, single-arm, phase II study of patients with unresectable locally advanced or metastatic hereditary MTC.

\section{METHODS}

\section{Patients}

Adult patients who had unresectable, locally advanced or metastatic MTC with a confirmed clinical diagnosis of MEN2A, MEN2B, or FMTC and a germline RET mutation were eligible. Other key inclusion criteria included the presence of at least one measurable lesion according to Response Evaluation Criteria in Solid Tumors (RECIST) guidelines ${ }^{14}$; WHO performance status of 0 to 2; and adequate cardiac, hematopoietic, hepatic, and renal function. Patients with brain metastases were eligible if they were treated with radiation therapy at least 4 weeks before entry and were clinically stable without corticosteroid treatment for 1 week. Patients were ineligible if they had received prior chemotherapy and/or radiation therapy within 4 weeks before the initiation of the study therapy. Patients were excluded if there was evidence of pheochromocytoma on the basis of elevated 24-hour urinary catecholamine levels. The protocol was approved by all relevant institutional ethical committees and was conducted in accordance with the Declaration of Helsinki. All patients provided written informed consent.

\section{Study Design}

This was an open-label, phase II study conducted at seven centers. Patients received once-daily oral doses of vandetanib $300 \mathrm{mg}$ until disease progression, unacceptable toxicity, or withdrawal of consent occurred. The primary end point was objective response rate (by RECIST). Additional assessments included the duration of response; disease control; progression-free survival (PFS); safety and tolerability; and changes in serum levels of the polypeptide, calcitonin, and the glycoprotein, carcinoembryonic antigen (CEA), which are secreted by MTC cells.

\section{Objective Tumor Response}

Determination of objective response was based on investigator-reviewed computed tomography (CT) or magnetic resonance imaging (MRI) scans obtained at initial screening and at 12-week intervals during treatment until objective disease progression or withdrawal occurred. An objective complete or partial response (by RECIST) was confirmed by repeat imaging not less than 4 weeks from the initial response. The designation of stable disease required at least one assessment of stable disease or an unconfirmed objective response at least 8 weeks after starting treatment. An independent central review was performed of all scans used for RECIST assessment at each site.

\section{Safety and Tolerability}

Throughout the study, safety was assessed by monitoring and recording of adverse events, 12-lead ECG parameters, vital signs, clinical chemistry, hematology, and urinalysis. Adverse events were graded according to the National Cancer Institute Common Terminology Criteria for Adverse Events (CTCAE, version 3). Vandetanib dose adjustments were made as defined in the protocol for grade 3 or 4 toxicity. Unless additional ECGs were clinically indicated, 12-lead ECGs were performed during screening, weekly for the first 8 weeks, and then every 12 weeks thereafter. Assessment of the corrected QT
(QTc) interval by using Bazett's correction was evaluated at each site and by a central reviewer. Prolongation was defined as a single measurement of $\geq 550$ milliseconds or an increase of $\geq 100$ milliseconds from baseline; or as two consecutive measurements (within 48 hours of each other) that were $\geq 500$ milliseconds but less than 550 milliseconds, or an increase of $\geq 60$ milliseconds but less than 100 milliseconds from baseline to a value $\geq 480$ milliseconds.

\section{Measurement of Serum Tumor Markers}

Blood samples for calcitonin analysis were collected at baseline, at day 5 , weekly for the first 8 weeks, and then monthly thereafter. Serum calcitonin levels were determined by an immunochemiluminescent method (Quest Diagnostics Nichols Institute, San Juan Capistrano, CA). Blood samples for CEA analysis were collected at baseline, at day 28 , and then monthly thereafter. Serum CEA measurements were performed by using a commercially available microparticle enzyme immunoassay (Abbott Diagnostics, Abbott Park, IL). For calcitonin and CEA analysis, best response for a patient was calculated from assessments at baseline and during treatment and was defined as follows: complete response, complete normalization of serum levels after treatment; partial response, $50 \%$ or greater decrease from baseline levels maintained during a minimum of 4 weeks; stable disease, between $20 \%$ and $-50 \%$ change from baseline levels maintained for at least 4 weeks; progressive disease, $20 \%$ or greater increase from baseline maintained for at least 4 weeks.

\section{Statistical Analysis}

A two-stage study design was employed to evaluate the null hypothesis that the true objective response rate (ORR) was less than $20 \%$ and the alternative hypothesis that the ORR was $\geq 20 \%$. There was a target accrual of 15 patients in the first stage, with an additional 15 patients enrolled if at least one response was observed. All patients who received one dose or more of vandetanib were included in the safety and efficacy analyses. Estimates of PFS (ie, time from first dose of study drug to progression or death by any cause within 3 months of last tumor assessment) with associated 95\% CIs were obtained by using the Kaplan-Meier method. Median duration of response (ie, interval between the onset of objective response and evidence of progressive disease) and disease control rate (ie, patients who had a best response of complete response or partial response or who had stable disease for $\geq 24$ weeks) were summarized with associated $95 \%$ CIs.

\section{RESULTS}

\section{Patients}

Between November 12, 2004 and August 15, 2006, a total of 30 patients were enrolled and started treatment with vandetanib. Seventeen patients were continuing treatment at the time of data cutoff (February 22, 2008); four of these patients were assessed to have disease progression by RECIST but continued to receive treatment because their physicians thought they were receiving clinical benefits. The remaining patients discontinued vandetanib because of adverse events $(n=7)$, disease progression $(n=4)$, or withdrawal of consent $(n=2)$. The demographics and baseline characteristics of study patients are listed in Table 1. The majority of patients had MEN2A. At study entry, 29 patients had distant metastases, including sites of liver (80\%), lymph nodes (70\%), and lung (63\%).

\section{Efficacy}

Twenty-nine patients were assessable for objective tumor response, but all 30 patients were included in the intent-to-treat analysis (Table 2). According to investigator assessments, a confirmed objective partial response was achieved in six patients $(20 \%)$. The median duration of response at data cutoff was 10.2 months (range, 1.9 to 16.9 months; $95 \%$ CI, 8.0 to 13.2 months), and three patients developed progressive disease subsequent to confirmation (one each at 10.6 months, 27.3 months, and 27.9 months). Durable stable disease (ie, 
Table 1. Demographic and Baseline Clinical Characteristics of Patients

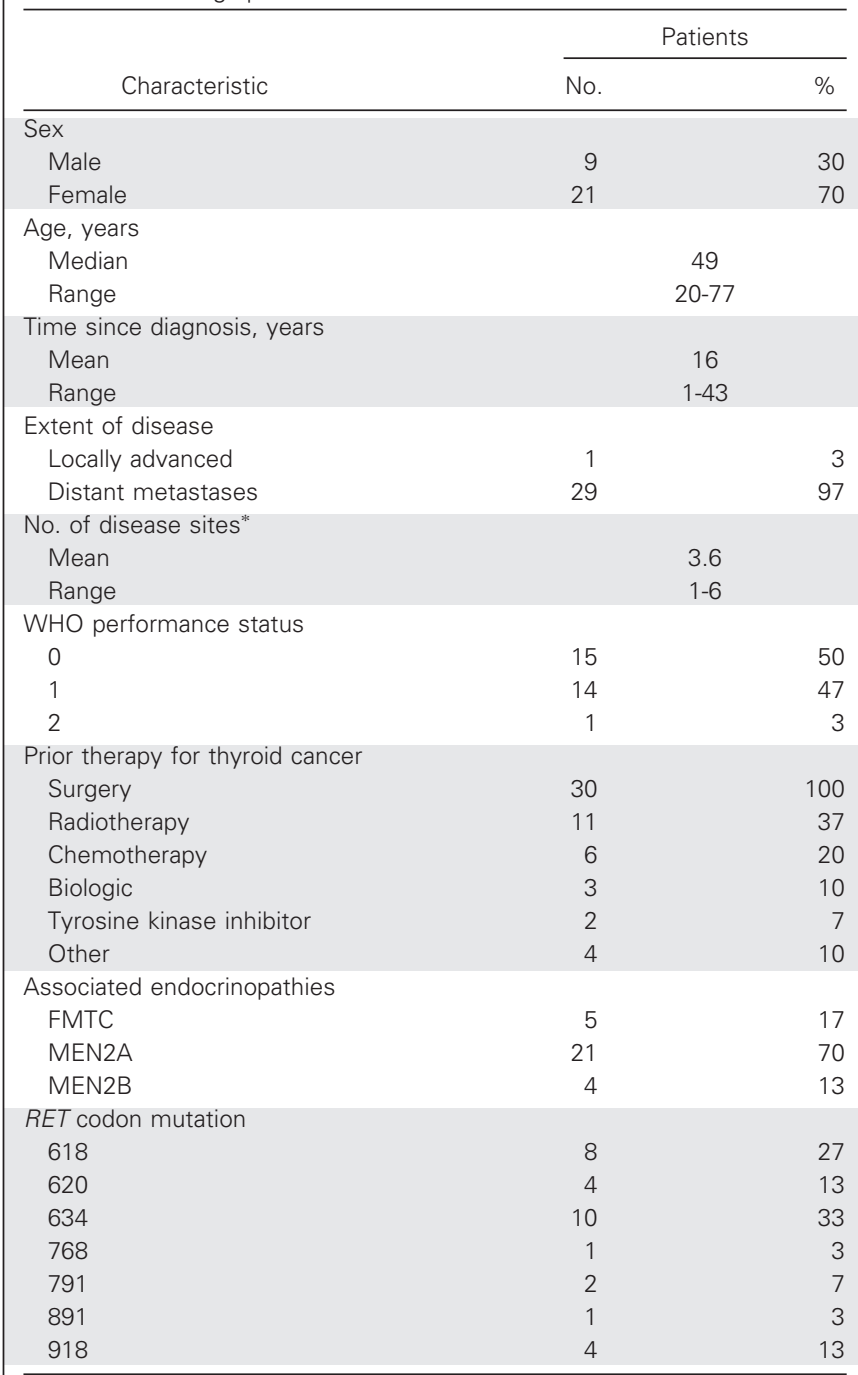

Abbreviations: FMTC, familial medullary thyroid cancer; MEN, multiple endocrine neoplasia; RET, rearranged during transfection.

*Including primary tumor.

disease that was stable for $\geq 24$ weeks) was reported in 16 patients (53\%). Disease control (ie, confirmed partial response and stable disease for $\geq 24$ weeks), therefore, was observed in 22 patients (73\%). Six patients had a best response of stable disease for less than 24 weeks, one patient had progressive disease as a best response, and one patient was not evaluable for objective tumor response. This last patient entered the study with an elevated creatinine and BUN at baseline: $150.3 \mu \mathrm{mol} / \mathrm{L}$ (ie, CTCAE grade 1 ) and $7.9 \mathrm{mmol} / \mathrm{L}$, respectively (increasing to $406.6 \mu \mathrm{mol} / \mathrm{L}$ [ie, CTCAE grade 3] and 14.6 $\mathrm{mmol} / \mathrm{L}$, respectively, at week 5). The patient was terminated from study shortly after beginning vandetanib treatment and before any follow-up scans were performed.

As shown in Figure 1, 25 patients (83\%) experienced some reduction in tumor size during vandetanib treatment. There was no apparent association between RET germline mutation and response to vandetanib treatment (Table 3 ). In addition to the six patients with confirmed partial responses, five patients had unconfirmed partial responses. One of these patients had a single RECIST assessment that
Table 2. Tumor Response According to Investigator Review by RECIST

\begin{tabular}{|c|c|c|}
\hline \multirow[b]{2}{*}{ Best RECIST Response } & \multicolumn{2}{|c|}{$\begin{array}{l}\text { All Patients } \\
(\mathrm{N}=30)\end{array}$} \\
\hline & No. & $\%$ \\
\hline CR & 0 & \\
\hline Confirmed PR & 6 & 20 \\
\hline$S D \geq 24$ weeks & 16 & 53 \\
\hline$S D \geq 8$ weeks but $<24$ weeks & 6 & 20 \\
\hline Progressive disease & 1 & 3 \\
\hline Not evaluable & 1 & 3 \\
\hline \multicolumn{3}{|l|}{ Objective response rate $(C R+P R)$} \\
\hline$\%$ & \multirow{2}{*}{\multicolumn{2}{|c|}{$\begin{array}{l}20 \\
8-39\end{array}$}} \\
\hline $95 \% \mathrm{Cl}$ & & \\
\hline \multicolumn{3}{|c|}{ Disease control rate (PR $+S D \geq 24$ weeks) } \\
\hline$\%$ & \multirow{2}{*}{\multicolumn{2}{|c|}{$\begin{array}{l}73 \\
54-88\end{array}$}} \\
\hline $95 \% \mathrm{Cl}$ & & \\
\hline \multicolumn{3}{|l|}{ Duration of response, months* } \\
\hline Median & \multicolumn{2}{|c|}{10.2} \\
\hline Range & \multicolumn{2}{|c|}{$1.9-16.9$} \\
\hline \multicolumn{3}{|l|}{ Time to first response, months } \\
\hline Median & \multirow{2}{*}{\multicolumn{2}{|c|}{$\begin{array}{c}7.1 \\
2.6-16.4\end{array}$}} \\
\hline Range & & \\
\hline \multicolumn{3}{|l|}{ PFS, months } \\
\hline Median & \multicolumn{2}{|c|}{27.9} \\
\hline $95 \% \mathrm{Cl}$ & \multicolumn{2}{|c|}{ 19.4-NE } \\
\hline
\end{tabular}

Abbreviations: RECIST, Response Evaluation Criteria in Solid Tumors; CR, complete response; $\mathrm{PR}$, partial response; $\mathrm{SD}$, stable disease; PFS, progressionfree survival; NE, not estimable.

*From first response to progression or death.

demonstrated partial response, but that patient was deemed to have progressive disease at the next assessment. The partial responses in the other four patients occurred late in the treatment course, at the last assessment before data cutoff.

At the time of data cutoff, the estimated median PFS was 27.9 months (95\% CI, 19.4 to not estimable); eight patients (27\%) had RECIST-defined progression, 20 patients $(67 \%)$ had no progression and were still alive at the time of analysis, and two patients had died more than 3 months after their last RECIST assessments (one patient of cardiac failure and the other of colon cancer).

The best response results that were based on independent central review were similar to those obtained from the investigational site,

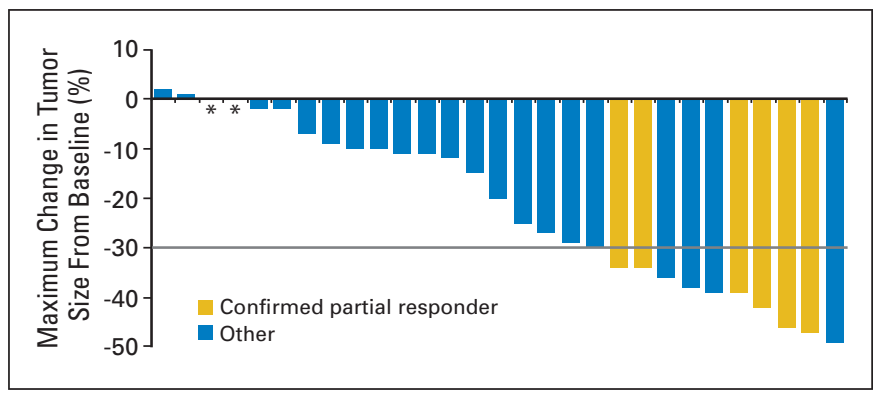

Fig 1. Maximum reduction from baseline (or smallest increase from baseline for patients with no reductions) in the sum of the longest diameters of target lesions. The change from baseline in tumor measurement as assessed by investigator review is shown for 29 patients; one non-evaluable patient is not shown. Asterisk indicates patients with a best response of $0 \%$ change from baseline (stable disease $\geq 8$ weeks but $<24$ weeks, $n=1$; stable disease $\geq 24$ weeks, $n=1$ ). Gray line represents the threshold for partial response ( $>30 \%$ reduction from baseline sum of longest diameters). Target lesions were defined according to the Response Evaluation Criteria in Solid Tumors (RECIST). 


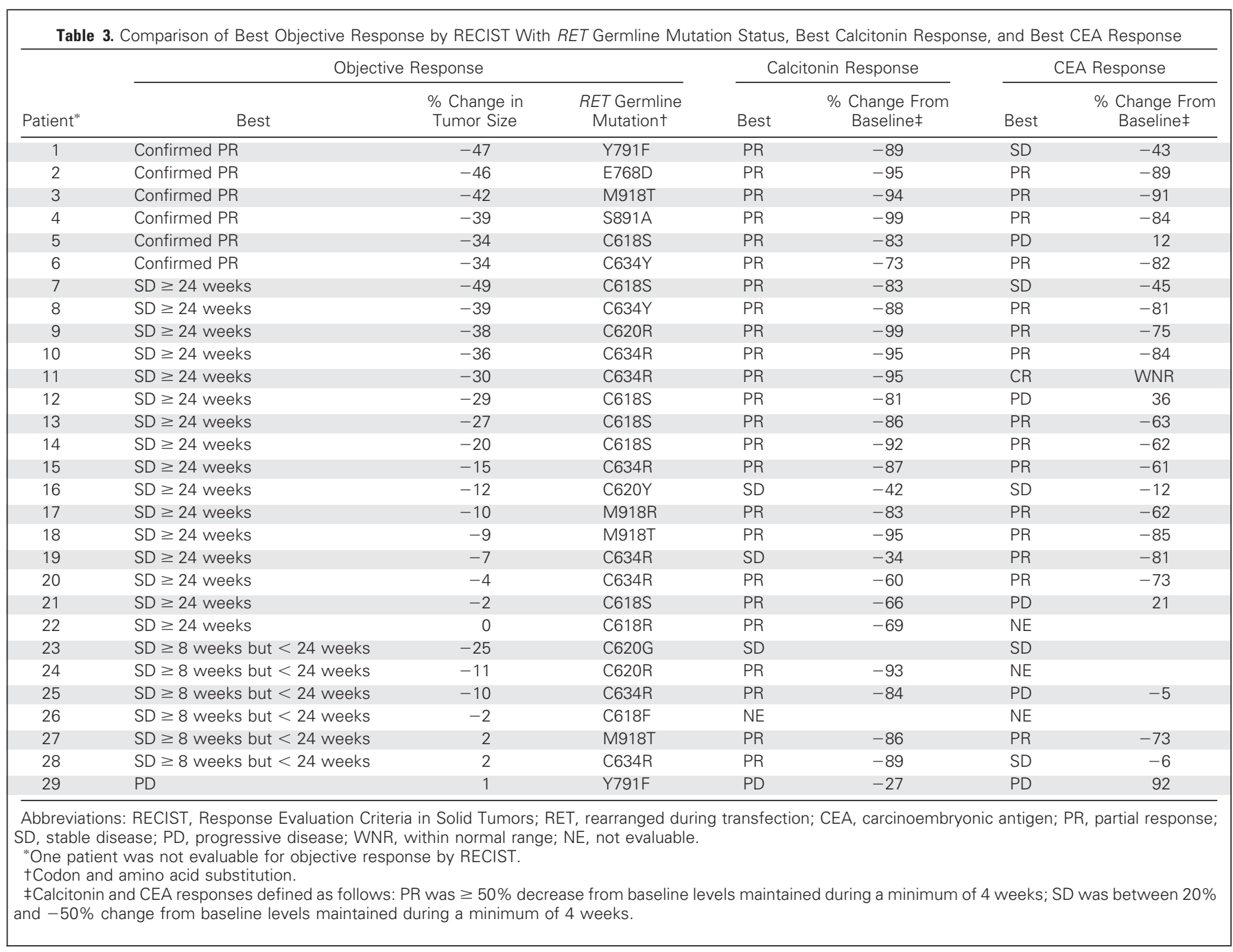

although there was discordance in eight patients. On the site review, six patients had a confirmed partial response, 22 patients had stable disease, one patient had progressive disease, and one patient was not evaluable. On central review, five patients had a confirmed partial response, 22 patients had stable disease, one patient had progressive disease, and two patients were not evaluable. The differences between the central and site review included four patients with confirmed partial responses on site review who had stable disease on central review; three patients with stable disease on site review who had a confirmed partial response on central review; and one patient with stable disease on site review who was classified as not evaluable on central review. These discordances had no adverse impact on the estimated median PFS (27.9 months on the basis of site review $v 34.7$ months on the basis of central review).

\section{Serum Tumor Markers}

Decreases from baseline in serum calcitonin and CEA levels, which fulfilled criteria for a partial response, occurred in 24 patients (80\%) and 16 patients (53\%), respectively, and exceeded the partial response on the basis of scan reviews and RECIST criteria. All six patients with a confirmed partial response on imaging studies had calcitonin decreases ranging from $73 \%$ to
$99 \%$, and four patients had CEA decreases ranging from $82 \%$ to 91\% (Table 3).

\section{Safety and Tolerability}

The median duration of vandetanib therapy was 18.8 months (range, 0.6 to 38.4 months). Seven patients (23\%) discontinued treatment because of adverse events, five of which were considered by the investigator to be vandetanib related (including hemorrhagic diarrhea, nausea, increased blood creatinine and BUN, acne, and asymptomatic QTc prolongation). In two of the seven patients, the adverse events were thought to be unrelated to vandetanib. In one patient with histologic evidence of MTC hepatic metastases, gastrointestinal obstruction that was thought to be due to diverticulitis was found at operation to be due to colon cancer. The second patient had been treated with dacarbazine, streptozotocin, and doxorubicin before study entry and subsequently had to be withdrawn because of heart failure and a pleural effusion. Vandetanib dosing was reduced or interrupted in 24 patients because of adverse events, most commonly diarrhea $(n=7)$. Ten patients had dose reductions without interrupting administration of vandetanib, and 11 patients had a temporary interruption of vandetanib administration and then had subsequent dose reduction. Among the 24 patients who required dose reduction 
or interruption, 21 patients had a combination of dose reduction and dose interruption (some patients required more that a single dose reduction), and three patients had a dose interruption without dose reduction. For patients who required dose reductions, vandetanib was decreased to $200 \mathrm{mg}(\mathrm{n}=18), 150 \mathrm{mg}(\mathrm{n}=1), 100 \mathrm{mg}(\mathrm{n}=7)$, or 50 $\mathrm{mg}(\mathrm{n}=2)$. The median time to first dose reduction was 4.9 months (95\% CI, 2.7 to 8.4 months).

Adverse events were predominantly grade 1 or 2 , and the most common events included diarrhea, fatigue, rash, and nausea (Table 4). The most common grade 3 adverse events were QTc prolongation $(\mathrm{n}=6)$ and diarrhea, nausea, and hypertension $(\mathrm{n}=3$ each). There were grade 4 adverse events of azotemia or muscle weakness $(n=1$ each), which were not considered by the investigator to be related to vandetanib. All of these events were managed with dose interruptions or reductions. Other adverse events included the following: mild visual disturbances (grade 1) in three patients due to vandetanib-related corneal changes (managed with vandetanib dose reductions) and hypophosphatemia (CTCAE grade 2, $\mathrm{n}=2$; CTCAE grade $3, \mathrm{n}=1$ ). Also, increases in blood pressure of greater than $30 \mathrm{mmHg}$ systolic higher than baseline were noted in 23 patients, but in no instance did it lead to permanent study discontinuation.

\section{DISCUSSION}

The majority of patients with hereditary or sporadic MTC are not cured by thyroidectomy, and there is a lack of effective therapy for patients with persistent or recurrent MTC. The pathogenetic role of RET mutations in hereditary MTC suggests that blockade of RET kinase signaling would be a rational therapeutic approach. Preclinical studies have demonstrated that vandetanib has strong inhibitory activity towards wild-type and constitutively active RET kinase. ${ }^{7}$ Furthermore, vandetanib has demonstrated antitumor effects in preclinical models of MTC. ${ }^{7,10,11}$

This study demonstrates that vandetanib has antitumor activity in patients with locally advanced or metastatic hereditary MTC, and

Table 4. Adverse Events Irrespective of Causality and Reported in 10 or More Patients

\begin{tabular}{|c|c|c|c|c|c|c|}
\hline \multirow[b]{3}{*}{ Event } & \multicolumn{6}{|c|}{ Patients by Event Grade } \\
\hline & \multicolumn{2}{|c|}{ Total } & \multicolumn{2}{|c|}{ Grades 1 or $2^{*}$} & \multicolumn{2}{|c|}{ Grade 3*† } \\
\hline & No. & $\%$ & No. & $\%$ & No. & $\%$ \\
\hline Diarrhea & 21 & 70 & 18 & 60 & 3 & 10 \\
\hline Rash & 20 & 67 & 19 & 63 & 1 & 3 \\
\hline Fatigue & 19 & 63 & 17 & 57 & 2 & 7 \\
\hline Nausea & 19 & 63 & 16 & 53 & 3 & 10 \\
\hline Headache & 14 & 47 & 13 & 43 & 1 & 3 \\
\hline Anorexia & 13 & 43 & 12 & 40 & 1 & 3 \\
\hline Vomiting & 12 & 40 & 10 & 33 & 2 & 7 \\
\hline Constipation & 11 & 37 & 10 & 33 & 1 & 3 \\
\hline Dysgeusia & 10 & 33 & 10 & 33 & NAł & \\
\hline Hypertension & 10 & 33 & 7 & 23 & 3 & 10 \\
\hline
\end{tabular}

Abbreviation: NA, not applicable.

*Graded according to National Cancer Institute Common Terminology Criteria of Adverse Events, version 3.

†Additional grades 3 to 4 adverse events are noted in the Results section. ¥No grade 3 dysgeusia in National Cancer institute Common Terminology Criteria of Adverse Events, version 3. confirmed objective partial responses were observed in six patients (20\%). The confirmed partial responses were durable (median duration, 10.2 months). An additional $53 \%$ of patients experienced stable disease for $\geq 24$ weeks, which yielded a disease control rate of $73 \%$, and the estimated median PFS was in excess of 27 months; these data all suggest clinically meaningful tumor control. An additional five patients (17\%) had unconfirmed partial responses, four of which occurred on the last assessment before data cutoff).

The determination of stable disease in patients with tumors such as MTC, which in some instances grow slowly, is difficult in the absence of documentation of progressive disease before study entry. Even though only five patients in this study had partial responses (by RECIST), it should be noted that 25 patients had a measurable reduction in the size of the tumor. In 21 patients, the reduction was greater than $10 \%$; in 14 patients, the reduction was greater than $20 \%$. Such reductions in size are rarely seen in untreated patients with advanced disease, and it is unlikely that the changes were caused by factors other than vandetanib.

The relative contribution of RET, VEGFR and EGFR inhibition to the observed clinical antitumor effects of vandetanib remain to be determined. Recent preliminary reports suggest that other tyrosine kinase inhibitors with activity against RET kinase have efficacy in MTC, ${ }^{15-17}$ but these compounds also inhibit VEGFR signaling pathways. It is unclear, therefore, which part of the spectrum of activity of these drugs is most dominant in producing the antitumor effects observed.

The accurate determination of tumor response poses challenges in patients with MTC and deserves additional study. Although overall response rates were similar on the basis of site and central reviews of the same radiographic images in this study, there were observer differences noted in eight patients. Multiple metastatic lesions are common in patients with MTC, and observer variability in the selection of target lesions may occur. There were striking reductions in serum levels of calcitonin and CEA in the majority of patients, most of which were of prolonged duration, but the correlation of marker response with response on the basis of scan assessment and RECIST criteria was imperfect. Serum calcitonin levels are used routinely as an indicator of MTC progression, as they are believed to vary directly with tumor mass. However, a recent preclinical study suggests that RET kinase inhibitors may inhibit calcitonin gene transcription and protein secretion in a manner dissociated from inhibition of tumor growth. ${ }^{18}$ It is unclear, therefore, whether calcitonin measurements will prove useful in evaluating tumor response to molecular therapeutics in patients with hereditary MTC or whether decreases in serum calcitonin will simply provide indirect evidence that RET kinase is being effectively inhibited. In this context, the decreases in serum CEA may serve as a more reliable marker of tumor response to vandetanib.

All patients in this study experienced at least one adverse event, but these adverse events were generally manageable with dose interruptions and reductions. The most common adverse events were diarrhea, fatigue, rash, and nausea; this is an adverse event profile consistent with previous vandetanib monotherapy studies. ${ }^{12,13}$ Diarrhea and rash in particular are typically associated with anti-EGFR therapy, and hypertension is associated with VEGF/VEGFR signaling inhibitors. ${ }^{19,20}$

Patients in this study received vandetanib at an initial oral dose of $300 \mathrm{mg} / \mathrm{d}$, which is considered to be the maximally tolerated daily dose. Since the initiation of this trial, a study using $100 \mathrm{mg} / \mathrm{d}$ as the 
initial dose of vandetanib in patients with advanced hereditary MTC has shown clinical activity. ${ }^{21}$ However, there has been no randomized trial directly comparing various vandetanib dosing regimens for patients with MTC.

In conclusion, vandetanib is a selective inhibitor of RET, VEGFR, and EGFR signaling. The incidence of partial responses, the prolonged duration of disease control, and the manageable adverse event profile observed in this study suggest that vandetanib may provide an effective therapeutic option in patients with locally advanced or hereditary MTC.

\section{AUTHORS' DISCLOSURES OF POTENTIAL CONFLICTS OF INTEREST}

Although all authors completed the disclosure declaration, the following author(s) indicated a financial or other interest that is relevant to the subject matter under consideration in this article. Certain relationships marked with a " $U$ " are those for which no compensation was received; those relationships marked with a " $C$ " were compensated. For a detailed description of the disclosure categories, or for more information about ASCO's conflict of interest policy, please refer to the Author Disclosure Declaration and the Disclosures of Potential Conflicts of Interest section in Information for Contributors.

Employment or Leadership Position: Annetta Krebs, AstraZeneca (C); James Vasselli, AstraZeneca (C) Consultant or Advisory Role: Samuel A. Wells Jr, AstraZeneca (C); Robert F. Gagel, Exelixis (C), Esai (U), Astra Zeneca (C), Amgen (C); Martin Schlumberger, Amgen (U), AstraZeneca (C), Bayer (C), Exelixis (U), Genzyme (C) Stock Ownership: Annetta
Krebs, AstraZeneca; James Vasselli, AstraZeneca Honoraria: Samuel A. Wells Jr, AstraZeneca; Robert F. Gagel, Exelixis, Astra Zeneca; Martin Schlumberger, AstraZeneca, Genzyme Research Funding: Robert F. Gagel, Astra Zeneca; Jeffrey Moley, Novartis; David Pfister, AstraZeneca; Martin Schlumberger, Amgen, AstraZeneca, Genzyme Expert Testimony: None Other Remuneration: Samuel A. Wells Jr, AstraZeneca

\section{AUTHOR CONTRIBUTIONS}

Conception and design: Samuel A. Wells Jr, Martin Schlumberger

Financial support: Martin Schlumberger

Administrative support: Samuel A. Wells Jr, Jeffrey Moley,

Martin Schlumberger

Provision of study materials or patients: Samuel A. Wells Jr, Jessica E. Gosnell, Robert F. Gagel, Jeffrey Moley, David Pfister, Julie A. Sosa, Michael Skinner, Martin Schlumberger

Collection and assembly of data: Samuel A. Wells Jr, Robert F. Gagel, Jeffrey Moley, David Pfister, Michael Skinner, James Vasselli,

Martin Schlumberger

Data analysis and interpretation: Samuel A. Wells Jr, Jessica E. Gosnell, Robert F. Gagel, David Pfister, Julie A. Sosa, Michael Skinner, Annetta Krebs, James Vasselli, Martin Schlumberger

Manuscript writing: Samuel A. Wells Jr, Robert F. Gagel, David Pfister, Julie A. Sosa, Annetta Krebs, James Vasselli, Martin Schlumberger Final approval of manuscript: Samuel A. Wells Jr, Jessica E. Gosnell, Robert F. Gagel, Jeffrey Moley, David Pfister, Julie A. Sosa, Michael Skinner, Annetta Krebs, James Vasselli, Martin Schlumberger

\section{REFERENCES}

1. Schlumberger M, Carlomagno F, Baudin E, et al: New therapeutic approaches to treat medullary thyroid carcinoma. Nat Clin Pract Endocrinol Metab 4:22-32, 2008

2. Kouvaraki MA, Shapiro SE, Perrier ND, et al: RET proto-oncogene: A review and update of genotype-phenotype correlations in hereditary medullary thyroid cancer and associated endocrine tumors. Thyroid 15:531-544, 2005

3. Roman S, Lin R, Sosa JA: Prognosis of medullary thyroid carcinoma: Demographic, clinical, and pathologic predictors of survival in 1252 cases. Cancer 107:2134-2142, 2006

4. Donis-Keller H, Dou S, Chi D, et al: Mutations in the RET proto-oncogene are associated with MEN 2A and FMTC. Hum Mol Genet 2:851-856, 1993

5. Mulligan LM, Kwok JB, Healey CS, et al: Germ-line mutations of the RET proto-oncogene in multiple endocrine neoplasia type 2A. Nature 363 458-460, 1993

6. Hofstra RM, Landsvater RM, Ceccherini I, et al: A mutation in the RET proto-oncogene associated with multiple endocrine neoplasia type $2 \mathrm{~B}$ and sporadic medullary thyroid carcinoma. Nature 367:375376, 1994

7. Carlomagno $F$, Vitagliano $D$, Guida $T$, et al: ZD6474, an orally available inhibitor of KDR tyrosine kinase activity, efficiently blocks oncogenic RET kinases. Cancer Res 62:7284-7290, 2002
8. Bunone $G$, Vigneri $P$, Mariani L, et al: Expression of angiogenesis stimulators and inhibitors in human thyroid tumors and correlation with clinical pathological features. Am J Pathol 155:1967-1976, 1999

9. Wedge SR, Ogilvie DJ, Dukes $M$, et al: ZD6474 inhibits vascular endothelial growth factor signaling, angiogenesis, and tumor growth following oral administration. Cancer Res 62:4645-4655, 2002

10. Santoro M, Carlomagno F: Drug insight: Small molecule inhibitors of protein kinases in the treatment of thyroid cancer. Nat Clin Pract Endocrinol Metab 2:42-52, 2006

11. Vidal M, Wells $S$, Ryan $A$, et al: ZD6474 suppresses oncogenic RET isoforms in a Drosophila model for type 2 multiple endocrine neoplasia syndromes and papillary thyroid carcinoma. Cancer Res 65:3538-3541, 2005

12. Holden $S N$, Eckhardt $S G$, Basser $R$, et al: Clinical evaluation of ZD6474, an orally active inhibitor of VEGF and EGF receptor signaling, in patients with solid, malignant tumors. Ann Oncol 16:13911397, 2005

13. Tamura $T$, Minami $H$, Yamada $Y$, et al: A Phase I dose-escalation study of ZD6474 in Japanese patients with solid, malignant tumors. J Thorac Oncol 1:1002-1009, 2006

14. Therasse $P$, Arbuck SG, Eisenhauer EA, et al: New guidelines to evaluate the response to treatment in solid tumors: European Organization for Research and Treatment of Cancer, National Cancer Institute of the United States, National Cancer
Institute of Canada. J Natl Cancer Inst 92:205-216 2000

15. Schlumberger, M. Elisei, R, Bastholt, L, et al: Phase II study of safety and efficacy of motesanib in patients with progressive or symptomatic, advanced or metastatic medullary thyroid carcinoma. J Clin Oncol 27:3794-3801, 2009

16. Kober F, Hermann M, Handler A, et al: Effect of sorafenib in symptomatic metastatic medullary thyroid cancer. J Clin Oncol 25:617s, 2007 (suppl; abstr 14065)

17. Salgia $R$, Sherman $S$, Hong $D$, et al: A phase study of XL184, a RET, VEGFR2 and MET kinase inhibitor in patients with advanced malignancies, including patients with medullary thyroid cancer. J Clin Oncol 26:158s, 2008 (suppl; abstr 3522)

18. Akeno-Stuart N, Croyle M, Knauf JA, et al: The RET kinase inhibitor NVP-AST487 blocks growth and calcitonin gene expression through distinct mechanisms in medullary thyroid cancer cells. Cancer Res 67:6956-6964, 2007

19. Izzedine $H$, Rixe $O$, Billemont $B$, et al: Angiogenesis inhibitor therapies: Focus on kidney toxicity and hypertension. Am J Kidney Dis 50:203-218, 2007

20. Herbst RS: Toxicities of antiangiogenic therapy in non-small-cell lung cancer. Clin Lung Cancer 8:S23-S30, 2006 (suppl 1)

21. Haddad RI, Krebs AD, Vasselli J, et al: A phase Il open-label study of vandetanib in patients with locally advanced or metastatic hereditary medullary thyroid cancer. J Clin Oncol 26:322s, 2008 (suppl; abstr 6024) 